Our case highlights the importance of timely recognition of a falsepositive result for SARS-CoV-2 when chest CT findings do not conform to the typical changes of COVID-19 pneumonia. From the epidemic control perspective, it is imperative to isolate individuals with suspected cases of COVID-19 to protect the uninfected population. However, the suspected cases should not be housed with confirmed cases of COVID-19 to prevent the spread of this disease.

Acknowledgments. We are appreciated with Dr.Chuyi Cai and Prof. Getu Zhaori for their kind help to translate and polish some contents of the manuscript.

Financial support. This work was supported by Key Research Foundation of Hwa Mei Hospital, University of Chinese Academy of Sciences, China (Grant No. 2020HMZD19, 2020HMZD20).
Conflicts of interest. All authors report no conflicts of interest relevant to this article.

\section{References}

1. Bernheim A, Mei X, Huang M, et al. Chest CT findings in coronavirus disease-19 (COVID-19): relationship to duration of infection. Radiology February 20, 2020 [Epub ahead of print]. doi: 10.1148/radiol.2020 200463.

2. Chua F, James DA, Desai SR, et al. The role of CT in case ascertainment and management of COVID-19 pneumonia in the UK: insights from highincidence regions. Lancet Infect Dis March 25, 2020 [Epub ahead of print]. doi: 10.1016/S2213-2600(20)30132-6.

3. Yan G, Lee CK, Lam LTM, et al. Covert COVID-19 and false-positive dengue serology in Singapore. Lancet Infect Dis 2020 March 4 [Epub ahead of print]. doi: 10.1016/S1473-3099(20)30158-4.

\title{
A dynamic residential community-based quarantine strategy: China's experience in fighting COVID-19
}

\author{
Yan Guo PhD ${ }^{1,2}$, Yiran Li MS², Aliza Monroe-Wise MD, MSc 3 , Sai-Ching Jim Yeung MD, $\mathrm{PhD}^{4}$ and Yixiang Huang PhD ${ }^{5,1}$ (i) \\ ${ }^{1}$ Sun Yat-sen Global Health Institute, Sun Yat-sen University, Guangzhou, China, ${ }^{2}$ Department of Medical Statistics, School of Public Health, Sun Yat-sen \\ University, Guangzhou, China, ${ }^{3}$ Department of Global Health, University of Washington, Seattle, Washington, United States, ${ }^{4}$ Department of Emergency Medicine, \\ The University of Texas MD Anderson Cancer Center, Houston, Texas, United States and ${ }^{5}$ Department of Health Policy and Management, School of Public Health, \\ Sun Yat-sen University, Guangzhou, China
}

To the Editor-As the global COVID-19 pandemic progresses, many countries face major public health emergencies. The number of active COVID-19 cases worldwide is $>1.69$ million as of April $12,2020 .{ }^{1} \mathrm{~W}$ ith rapidly increasing new cases, healthcare systems are at the brink of collapse in some regions. To reduce the burden on health systems, public health strategies should be adopted to control the source of infection, to cut off transmission routes, and to protect vulnerable populations. One strategy that was effective in China in controlling the COVID-19 epidemic was the successful implementation of a nationwide, community-based, dynamic quarantine strategy. ${ }^{2}$ From Late January to March 18, 2020, the main purpose was to prevent COVID-19 from spreading in China. Afterward, the focus turned to the prevention of imported cases.

At the beginning of China's quarantine, residents were required to stay home. When necessary, they were required to use an electronic pass system with traceable personal information to gain entry to residential areas, work places, and public transportation, and body temperature was screened by thermal scanning at the entrances. ${ }^{3}$ Once new cases were identified, health professionals and volunteers followed-up, treated, and isolated the patient and those in close contact. From prior experience with SARS, most Chinese citizens understood the importance of the quarantine and were invested in its success. As the epidemic came under better control, community-level management was strengthened and upgraded. It also became more dynamic, allowing movement

Author for correspondence: Yixiang Huang, E-mail: huangyx@mail.sysu.edu.cn

Cite this article: Guo Y, et al. (2020). A dynamic residential community-based quarantine strategy: China's experience in fighting COVID-19. Infection Control \& Hospital Epidemiology, 41: 1363-1364, https://doi.org/10.1017/ice.2020.172 and return to work to minimize the effects on people's lives and businesses while still monitoring movements and health status. ${ }^{4}$

Experience can be drawn from China's quarantine strategy. First, the quarantine strategy was strictly implemented nationwide at a community level. ${ }^{5}$ Strong governmental support is required to strengthen the community, and training is needed to foster policy implementation. Second, the quarantine strategy was dynamic and was adjustable based on the evolving situations, from stay-home quarantine to movement with updated health monitoring. Third, a large team of professional and technical support traced, identified, treated, and isolated patients and their close contacts. These strategies ensured early diagnosis and treatment, thus bringing the COVID-19 pandemic under control in China.

As the COVID-19 pandemic continues to take a massive toll on the healthcare systems and economies of countries worldwide, China's experience in its fight against this novel virus can be of great value to other countries. A practical attitude of learning by doing and responsiveness among government officials and the general population must be fostered.

\section{Acknowledgments.}

Financial support. This work was supported by the National Social Science Fund of China (grant no. 18BGL218) and the China Medical Board (grant no. 17-271).

Conflicts of interest. All the authors declare no conflicts of interest.

\section{References}

1. Coronavirus disease 2019 (COVID-19) situation report, 2020. World Health Organization website. https:/www.who.int/emergencies/diseases/novelcoronavirus-2019/situation-reports. Published 2020. Accessed April 13, 2020. 
2. Wu Z, McGoogan JM. Characteristics of and important lessons from the coronavirus disease 2019 (COVID-19) outbreak in China: summary of a report of 72,314 cases from the Chinese Center for Disease Control and Prevention. JAMA 2020;323:1239-1242.

3. Beijing Youth Daily. Electronic pass system: an innovative form of social management, 2020. http://opinion.people.com.cn/n1/2020/0218/c100331591658.html. Published 2020. Accessed April 1, 2020.
4. Changjiang Internet. Many Chinese cities have recognized health QR code of the people from Hubei, 2020. http://news.cjn.cn/sywh/202003/t3598691. htm. Published 2020. Accessed April 1, 2020.

5. Tang Y. Challenges and responses of community governance in the prevention and control of novel coronary pneumonia: from perspectives of urban-rural planning and public health [in Chinese]. Nanjing Social Science 2020:8-14+27.

\title{
Extended use or reuse of N95 respirators during COVID-19 pandemic: An overview of national regulatory authority recommendations
}

\author{
Leticia Mitiko Kobayashi BSc ${ }^{1}$, Bianca Ramos Marins $\mathrm{PhD}^{2}$, Patrícia Cristina dos Santos Costa $\mathrm{PhD}^{3}$, \\ Hugo Perazzo PhD 4 (D) and Rodolfo Castro $\mathrm{PhD}^{5,6}$ (D) \\ ${ }^{1}$ Fundação Oswaldo Cruz, Instituto Nacional de Controle de Qualidade em Saúde, Rio de Janeiro, RJ, Brazil, ${ }^{2}$ Departamento de Saúde Coletiva, Universidade \\ Federal do Estado do Rio de Janeiro, Rio de Janeiro, RJ, Brazil, ${ }^{3}$ Departamento de Ciências Fisiológicas, Universidade Federal do Estado do Rio de Janeiro, Rio \\ de Janeiro, RJ, Brazil, ${ }^{4}$ Fundação Oswaldo Cruz, Instituto Nacional de Infectologia Evandro Chagas, Rio de Janeiro, RJ, Brazil, ${ }^{5}$ Fundação Oswaldo Cruz, Escola \\ Nacional de Saúde Pública Sergio Arouca, Rio de Janeiro, RJ, Brazil and ${ }^{6}$ Universidade Federal do Estado do Rio de Janeiro, Instituto de Saúde Coletiva, Rio de \\ Janeiro, RJ, Brazil
}

To the Editor-We read with great interest the article by Wang et $\mathrm{al}^{1}$ and the study by Cheng et $\mathrm{al}^{2}$ that highlighted the vital role of N95 respirators for preventing SARS-CoV-2 transmission and COVID-19 among healthcare workers (HCWs). The protective role of N95 respirators in other respiratory diseases could be translated to tackle the COVID-19 pandemic. ${ }^{1}$ Preliminary results in Hong Kong demonstrated that the use of N95 respirators for triage, for medical care of suspected or confirmed cases and during aerosol-generating procedures, drastically reduced COVID-19 infection among HCWs. ${ }^{2}$ We acknowledge that the effectiveness of N95 respirators to prevent SARS-CoV-2 transmission should be confirmed and their use in clinical practice should be supported during the COVID-19 pandemic. However, we are facing a scenario of global shortage in availability of personal protective equipment (PPE), including surgical masks and N95 respirators. ${ }^{3}$ Countries are in dispute over the insufficient number of manufacturers. With unfair markets and increasing prices, low- and middle-income countries are at risk of losing their ability to acquire PPE for their HCWs. Several studies have previously reported methods for PPE decontamination ${ }^{4}$ or reuse of N95 respirators. ${ }^{5}$

Globally, the discussion by health authorities regarding new approaches to managing the N95 respirator shortage is urgent. The extended use or reuse and/or implementation of decontamination methods of $\mathrm{N} 95$ respirators might be an alternative that can prevent SARS-CoV-2 transmission among HCWs during the COVID-19 pandemic. Therefore, we have summarized recommendations regarding the extended use or reuse of N95 respirators, and we provide an overview of published information by regulatory authorities, surveillance organizations, and ministries of health of several countries.

Author for correspondence: Rodolfo Castro, E-mail: rodolfoalcastro@gmail.com; rodolfo.castro@fiocruz.br.

Cite this article: Kobayashi LM, et al. (2020). Extended use or reuse of N95 respirators during COVID-19 pandemic: An overview of national regulatory authority recommendations. Infection Control \& Hospital Epidemiology, 41: 1364-1366, https:// doi.org/10.1017/ice.2020.173
Two researchers independently scrutinized the websites of the regulatory authorities of countries or regions and of ministries of health that a members or associates of the International Coalition of Medicines Regulatory Authorities (ICMRA). ${ }^{6}$ Following the screening of information up to April 10, 2020, information from each country or region was collected in an electronic database. We collected the date of publication and information and excerpts from the guidance document regarding the recommendations for extended and reuse of N95 masks or filtering face pieces (FFPs). Extended use was defined as use for longer periods without removing the respirator (eg, treating several patients or working for $>1$ shift without interruption), and reuse indicated that the respirator was removed, stored, and used at least 1 more time. ${ }^{7}$

Overall, 27 countries or regions were screened: 5 countries (19\%) only allowed extended use (Canada, France, Mexico, New Zealand and Sweden); 2 countries (7\%) mentioned only reuse (Germany and Netherlands); and 3 countries (11\%) recommended both strategies for rationing N95 respirators (Brazil, European region and the United States). No information was available on extended use or reuse of N95 respirators in the websites of 17 countries (63\%). Some countries (Germany and Netherlands) recommended specific methods for N95 respirators decontamination, and others (Europe and United States) mentioned several options, leaving the decision to health services managers (Table 1). The following decontamination methods were mentioned: dry heat in a drying cabinet at $65-70^{\circ} \mathrm{C}$ (Germany); vaporous hydrogen peroxide (Netherlands, Europe, and the United States); ultraviolet germicidal irradiation and moist heat (Europe and the United States). The maximum duration of extended used ranged from 4 hours (France, New Zealand, and Sweden) to 40 hours (Mexico), and the maximum number of cycles of decontamination ranged from 2 (Germany) to 5 (United States).

Emergency use authorization (EUA) by the US Food and Drug Administration (FDA) allows the use of unapproved medical products or unapproved use of approved medical products. Currently, PPE items, in vitro diagnostic tests, and ventilators 